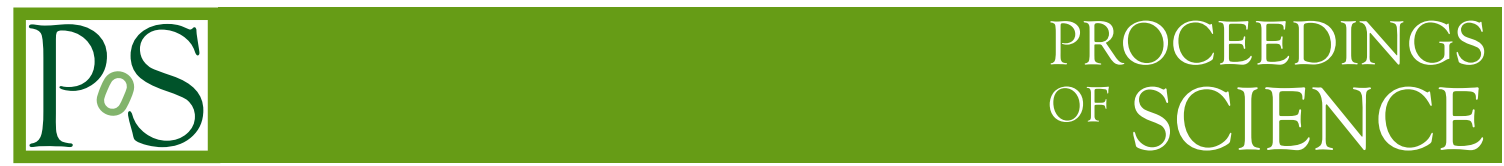

\title{
Leptogenesis constraints from flavour symmetry induced Lepton Mixing
}

\author{
Ivo de Medeiros Varzielas* \\ CFTP, Instituto Superior Técnico \\ E-mail: ivodcftp.ist.utl.pt
}

\begin{abstract}
In models with flavour symmetries added to the gauge group of the Standard Model the CPviolating asymmetry necessary for leptogenesis may be related with low-energy parameters. A particular case of interest is when the flavour symmetry produces an exact mass independent lepton mixing scheme, leading to a vanishing $\mathrm{CP}$-violating asymmetry. We present a modelindependent discussion that confirms this always occurs for unflavoured leptogenesis in type I see-saw scenarios.
\end{abstract}

35th International Conference of High Energy Physics

July 22-28, 2010

Paris, France

\footnotetext{
* Speaker.
} 


\section{Introduction}

This submission is based on [1] where a complete discussion with references is presented.

The smallness of neutrino masses can be understood within the see-saw mechanism where the Standard Model (SM) is extended by adding new heavy states. In type I see-saw the extra states are right-handed $(\mathrm{RH})$ neutrinos with large Majorana masses, and the light neutrino masses are generated via effective operators with the effective matrix given by $m_{v}=-m_{D} M_{R}^{-1} m_{D}^{T}$. In general $m_{D}$ and $M_{R}\left(M_{R}=M_{R}^{T}\right)$ are complex matrices. We have $\hat{m}_{D}=U_{L}^{\dagger} m_{D} U_{R}$ and $\hat{M}_{R}=V_{R}^{T} M_{R} V_{R}$, where the "^" denotes a diagonal matrix and $U_{L}, U_{R}, V_{R}$ are the diagonalising matrices. The unitary matrices $U_{\ell}, U_{\ell c}$ and $U_{v}$ diagonalise the charged lepton and neutrino mass matrices and the lepton mixing matrix is defined as $U=\left(U_{\ell}\right)^{\dagger} U_{v}$. The leptonic mixing indicated by data is approximated by the Tri-Bimaximal (TB) scheme, and this can be interpreted as a signal of an underlying flavour symmetry. If $m_{v}$ is exactly diagonalised by the TB mixing matrix $U_{T B}$ (in the basis with diagonal charged lepton mass matrix) we write $\hat{m}_{V}=D U_{T B}^{T} m_{v} U_{T B} D$, where $D=\operatorname{diag}\left(e^{i \varphi_{1}}, e^{i \varphi_{2}}, 1\right)$ accounts for the low-energy Majorana phases. The requirement of having exact TB diagonalisation can be expressed by rewriting the effective matrix as

$$
\hat{m}_{v}=-D\left(U_{T B}^{T} U_{L}\right) \hat{m}_{D}\left(U_{R}^{\dagger} V_{R}\right) \hat{M}_{R}^{-1}\left(V_{R}^{T} U_{R}^{*}\right) \hat{m}_{D}\left(U_{L}^{T} U_{T B}\right) D .
$$

The see-saw mechanism also contains the ingredients for leptogenesis. We discuss only "unflavoured" leptogenesis, as in the framework of flavour symmetry models predicting TB mixing the heavy singlet neutrinos typically have masses above $10^{13} \mathrm{GeV}$ and for $T \gtrsim 10^{12} \mathrm{GeV}$ lepton flavours are indistinguishable. In the standard thermal leptogenesis scenario singlet neutrinos $N_{\alpha}$ are produced by scattering processes after inflation. Subsequent out-of-equilibrium decays of these heavy states generate a $\mathrm{CP}$-violating asymmetry given by

$$
\varepsilon_{N_{\alpha}}=\frac{1}{4 v^{2} \pi\left(m_{D}^{R^{\dagger}} m_{D}^{R}\right)_{\alpha \alpha}} \sum_{\beta \neq \alpha} \operatorname{Im}\left[\left(\left(m_{D}^{R \dagger} m_{D}^{R}\right)_{\beta \alpha}\right)^{2}\right] f\left(z_{\beta}\right),
$$

where $z_{\beta}=M_{\beta}^{2} / M_{\alpha}^{2}, f\left(z_{\beta}\right)$ is the loop function, $m_{D}^{R} \equiv m_{D} V_{R}$ (i.e. in the basis where $M_{R}$ is diagonal).

The orthogonal complex matrix $R$ is defined by the Casas-Ibarra parametrisation

$$
R^{*}=\left(\hat{m}_{v}\right)^{-1 / 2} U^{T} m_{D}^{R}\left(\hat{M}_{R}\right)^{-1 / 2} .
$$

The matrix $R$ is very useful in expressing the CP-violating asymmetry. Considering for simplicity hierarchical RH neutrinos $\left(M_{1} \ll M_{2} \ll M_{3}\right)$, with $m_{j} \equiv\left(\hat{m}_{v}\right)_{j j}$, (1.2) can be rewritten as

$$
\varepsilon_{N_{\alpha}}=-\frac{3 M_{\alpha}}{8 \pi v^{2}} \frac{\operatorname{Im}\left[\sum_{j} m_{j}^{2} R_{j \alpha}^{2}\right]}{\sum_{j} m_{j}\left|R_{j \alpha}\right|^{2}} .
$$

Fixing the RH neutrino mass spectrum and low-energy observables, random values of $m_{D}^{R}$ correspond to random values of $R$. Leptogenesis is insensitive to low-energy lepton mixing and its viability is not related with any accidental mixing pattern considered. The asymmetry in (1.4) is determined by the values of the entries of $R$ which are arbitrary in the absence of any flavour symmetry, therefore $\varepsilon_{N_{a}} \neq 0$ in general. To illustrate this, consider a case where only $N_{1}$ decays are relevant and $R=R_{13}\left(\rho_{13}\right)$ with $\rho_{13}=\omega+i \sigma$ ( $R$ is a 13 rotation matrix with angle $\rho_{13}$ ) - Figure 1 shows that the asymmetry does not vanish in general (regardless of any low-energy mixing pattern). 


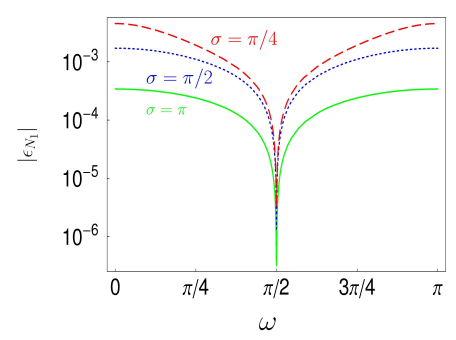

Figure 1: CP-violating asymmetry as a function of the angle $\omega$ for different values of $\sigma$.

\section{Leptogenesis with form-diagonalisability}

We consider now that an underlying flavour symmetry enforces an exact mixing pattern. In these cases, the mass matrices $m_{D}$ and $M_{R}$ (controlled by the transformation properties of $L_{i}$ and $N_{\alpha}$ under the symmetry group) are form-diagonalisable, i.e. their eigenvalues are completely independent from their diagonalising matrices, and the mixing matrix consists purely of numbers. The following proof holds under form-diagonalisability, but we assume TB mixing in particular (for definiteness). Vanishing off-diagonal elements of $\hat{m}_{v}$ in (1.1) can arise only if

$$
U_{T B}^{T} U_{L}=P_{L} O_{D_{i}} \quad \text { and } \quad U_{R}^{\dagger} V_{R}=O_{D_{i}}^{\dagger} P_{R} O_{R_{m}},
$$

where $P_{L, R}=\operatorname{diag}\left(e^{i \alpha_{1}^{R, L}}, e^{i \alpha_{2}^{R, L}}, e^{i \alpha_{3}^{R, L}}\right)$ whereas $O_{D_{i}}$ and $O_{R_{m}}$ are respectively unitary and orthogonal matrices that arbitrarily rotate the $i$ and $m$ degenerate eigenvalues of $m_{D}$ and $M_{R}$ such that if $m_{D}$ (or $M_{R}$ ) has no degenerate eigenvalues $O_{D_{i}}=\operatorname{diag}(1,1,1)=\operatorname{Id}$ (or $O_{R_{m}}=\mathrm{Id}$ ). The requirement of having canonical kinetic terms in addition to preserving the $m$-fold degeneracy of the RH neutrino mass matrix enforce $O_{R_{m}}$ to be real. Although $O_{D_{i}}$ and $O_{R_{m}}$ do not have any effect in (1.1) they affect the structure of $U_{L, R}$ and $V_{R}$ and correspondingly of $m_{D}$. $V_{R}$ can be defined in such a way that $\hat{M}_{R}$ is real, and the phases contained in $\hat{m}_{D}$, denoted by $\gamma_{i}$, must obey $\varphi_{i}+\alpha_{i}^{R}+\alpha_{i}^{L}+\gamma_{i}=2 k \pi$ and $\alpha_{3}^{R}+\alpha_{3}^{L}+\gamma_{3}=2 n \pi$. To understand the conditions given in (2.1) consider for simplicity the case without any degeneracy: $O_{D_{i}}=\mathrm{Id}$ and $O_{R_{m}}=$ Id. If the products $U_{T B}^{T} U_{L}$ and $U_{R}^{\dagger} V_{R}$ are not diagonal then the right-hand side of (1.1) is in general a matrix with entries that are linear combinations of the mass eigenvalues of $\hat{m}_{D}$ and of $\hat{M}_{R}$. In order to have the the off-diagonal entries of $\hat{m}_{v}$ vanish the respective linear combinations must all cancel out, but such cancellations correspond to having well-defined relationships between the eigenvalues of $\hat{m}_{D}$ and of $\hat{M}_{R}$ - it would require extreme fine-tuning of the masses. In order to avoid that one can instead consider that $U_{T B}^{T} U_{L}$ and $U_{R}^{\dagger} V_{R}$ are diagonal (or they must obey (2.1), in the general case with degeneracies).

Taking $O_{D_{i}}=\mathrm{Id}$ and $O_{R_{m}}=\mathrm{Id}$ for simplicity (the case with degeneracies is treated carefully in [1]), we write $\hat{m}_{D}=\operatorname{diag}\left(v_{1}, v_{2}, v_{3}\right)$ in the $\hat{m}_{D}$ basis, and in the $\hat{M}_{R}$ basis we have

$$
m_{D}^{R}=U_{T B} P_{L} \operatorname{diag}\left(v_{1}, v_{2}, v_{3}\right) P_{R},
$$

then redefining the $v_{i}$ by absorbing $P_{L}, P_{R}$ such that $m_{D}^{R}=U_{T B} \operatorname{diag}\left(v_{1}, v_{2}, v_{3}\right)$ we finally write

$$
m_{D}^{R}=U_{T B} P \hat{v},
$$

$\hat{v}=\operatorname{diag}\left(\left|v_{1}\right|,\left|v_{2}\right|,\left|v_{3}\right|\right)$ and the phases put into $P$. The type I see-saw formula of (1.1) becomes

$$
\hat{m}_{v}=-D U_{T B}^{T}\left(U_{T B} P \hat{v}\right) \hat{M}_{R}^{-1}\left(\hat{v} P U_{T B}^{T}\right) U_{T B} D=\left(\hat{v} \hat{M}_{R}^{-1 / 2} R^{\dagger}\right)\left(R^{*} \hat{M}_{R}^{-1 / 2} \hat{v}\right) .
$$


We used $D=P^{*} e^{-i \pi / 2}$, which is a consequence of our definition of $\hat{m}_{v}$ in (1.1), and we introduced the arbitrary orthogonal complex matrix $R$. From (2.4) we have that

$$
\hat{m}_{v}^{-1 / 2} \hat{v} \hat{M}_{R}^{-1 / 2} R^{\dagger}=\mathrm{Id} .
$$

As $R^{\dagger} R^{*}=R^{T} R=$ Id we arrive at the following expression with $R$ related to diagonal matrices

$$
R^{*}=\hat{m}_{v}^{-1 / 2} \hat{v} \hat{M}_{R}^{-1 / 2} \text {. }
$$

By comparing (2.6) with the parametrisation given in (1.3) we deduce that in the case of exact TB mixing the matrix $R$ is real and according to (1.4) the CP-violating asymmetry vanishes.

It is straightforward to check that the matrix $R$ still turns out to be real for other exact mixing schemes as long as their mixing matrix also consists purely of numbers (e.g. the Bi-maximal mixing scheme). Note also that the result is also generalisable to models with two or more than three RH neutrinos.

\section{Conclusions}

An important consequence of our proof is that if the TB mixing pattern is due to an underlying flavour symmetry in a type I see-saw scenario, the viability of leptogenesis depends upon possible departures from the exact pattern. In the context of models based on discrete flavour symmetries that predict TB mixing at leading order this is achieved through next to leading order corrections. Since the size of the deviations from TB mixing are not arbitrary (constrained by data), one might expect the CP-violating asymmetry to be constrained by low-energy observables.

We refer the interested reader to [1] for a more detailed discussion of this proof (as well as other related topics not discussed here). Importantly, [1] includes also several relevant references that have been omitted here due to the limited space.

\section{Acknowledgements}

We thank Luca Merlo for useful discussions. The work of IdMV was supported by FCT under the grant SFRH/BPD/35919/2007. The work of IdMV was partially supported by FCT through the projects POCI/81919/2007, CERN/FP/83503/2008 and CFTP-FCT UNIT 777 which are partially funded through POCTI (FEDER) and by the Marie Curie RTN MRTN-CT-2006-035505.

\section{References}

[1] D. Aristizabal Sierra, F. Bazzocchi, I. de Medeiros Varzielas, L. Merlo and S. Morisi, Tri-Bimaximal Lepton Mixing and Leptogenesis, Nucl. Phys. B 827 (2010) 34 [arXiv : 0908 . 0907 ] . 